\title{
Adsorption of Azobenzene on Hexagonal Boron Nitride Nanomesh Supported by $\mathrm{Rh}(111)$
}

Á. Szitás, R. Gubó, T. Pásztor, A. P. Farkas, T. Ajtai, L. Óvári,* K. Palotás,* A. Berkó, and Z. Kónya

Cite This: J. Phys. Chem. C 2020, 124, 14182-14194

Read Online

ABSTRACT: Adsorption properties of azobenzene, the prototypical molecular switch, were investigated on a hexagonal boron nitride (h-BN) monolayer ("nanomesh") prepared on $\mathrm{Rh}(111)$. The h-BN layer was produced by decomposing borazine $\left(\mathrm{B}_{3} \mathrm{~N}_{3} \mathrm{H}_{6}\right)$ at $1000-1050$ K. Temperature-programmed desorption (TPD) studies revealed that azobenzene molecules adsorbed on the "wire" and "pore" regions desorb at slightly different temperatures. Angle-resolved high-resolution electron energy loss spectroscopy (HREELS) measurements demonstrated that the first molecular layer is characterized predominantly by an adsorption geometry with the molecular plane parallel to the surface. Scanning tunneling microscopy (STM) indicated a clear preference for adsorption in the pores, manifesting a

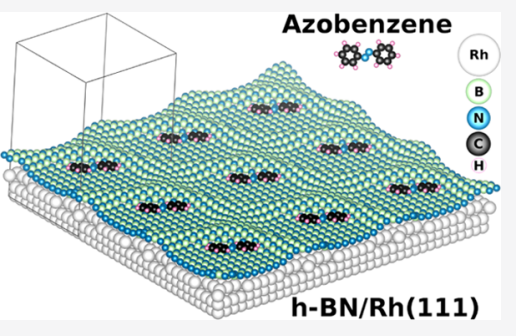
templating effect, but in some cases one-dimensional molecular stripes also form, implying attractive molecule-molecule interaction. Density functional theory (DFT) calculations provided further details regarding the adsorption energetics and bonding and confirmed the experimental findings that the molecules adsorb with the phenyl rings parallel to the surface, preferentially in the pores, and indicated also the presence of an attractive molecule-molecule interaction.

\section{INTRODUCTION}

The discovery of the fascinating properties of graphene, including its self-supporting nature and exceptional electronic properties, ${ }^{1,2}$ solicited vivid interest in other two-dimensional (2D) materials as well. ${ }^{3-7}$ Atomically thin hexagonal boron nitride (h-BN) has also been thoroughly investigated. ${ }^{5-7}$ It is isostructural and isoelectronic to graphene, but the difference in electronegativity results in an insulating character with an electronic band gap of about $6 \mathrm{eV}$. It has high chemical and thermal stability and also a high predicted thermal conductivity. ${ }^{8}$ Quite importantly, 2D hexagonal boron nitride is an excellent support and an ideal spacer for graphene-like nanoand optoelectronics. ${ }^{6,9}$ Besides, recent studies demonstrated that $\mathrm{h}-\mathrm{BN}$ alone or decorated with small $\mathrm{Au}$ nanoparticles is a highly selective catalyst in oxidative dehydrogenation or partial oxidation reactions. $^{10-12}$

h-BN monolayers have been "bottom-up" synthesized relatively easily on metal single-crystal surfaces of hexagonal symmetry $^{13-22}$ and also on rectangular ones like $\operatorname{Pd}(110),{ }^{23}$ as well as on $\operatorname{bcc}(110)$ surfaces, typically using borazine $\left(\mathrm{B}_{3} \mathrm{~N}_{3} \mathrm{H}_{6}\right)^{6,24}$ The morphology of the h-BN layer is determined by lattice mismatch and the strength of interaction between the nitride and the metal. The interaction with the elements of the copper group is weak, leading to the formation of a (nearly) planar h-BN monolayer. ${ }^{22,25-29}$ Ruthenium and rhodium bind the nitride strongly. Besides, the lattice mismatch between h-BN and the metal substrate is significant (7.0\% for $\mathrm{Rh}(111)$ and $8.2 \%$ for $\mathrm{Ru}(0001)$ ), leading to the formation of a periodically corrugated, continuous monolayer of h-BN ("nanomesh") on these metal substrates. ${ }^{16,19,28,30-32}$ The binding of h-BN to $\mathrm{Rh}(111)$ and $\mathrm{Ru}(0001)$ is the strongest with $\mathrm{N}$ atoms at the top position and $\mathrm{B}$ atoms in 3fold hollow sites, which is approximately fulfilled in the socalled "pore" regions. ${ }^{28}$ However, due to the lattice mismatch, there are regions ("wire") where this registry is not possible, resulting in weaker interactions and larger metal to h-BN distances. Interestingly, the nanomesh structure was observable on the $\mathrm{Au} / \mathrm{Rh}(111)$ surface alloys as well, up to relatively large $\mathrm{Au}$ contents $(\sim 0.9 \mathrm{ML})$; moreover, the pore diameter decreased significantly with the amount of $\mathrm{Au}$ in this range, allowing the tailored tuning of h-BN nanomesh morphology with the gold content. ${ }^{33}$ The periodically undulating h-BN monolayer formed on $\mathrm{Rh}(111)$ can be used as a template for the preparation of metal nanoparticles and related heterostructures. $^{33-35}$

The adsorption of (large) organic molecules on metalsupported h-BN monolayers has been intensely studied in the context of electronic decoupling, site-selective adsorption, orientational switching as a function of coverage, site-selective gating and charging, tip-gated charging, and even intercalation. Possible applications can be diverse, ranging from molecular electronics, heterogeneous catalysis, sensing, to light harvesting. The nanomesh superlattice, specifically, proved to be applicable as a template for the adsorption of organic

Received: February 27, 2020

Revised: June 3, 2020

Published: June 3, 2020 
molecules, as has already been pointed out for the $\mathrm{C}_{60}$ monolayer on $\mathrm{h}-\mathrm{BN} / \mathrm{Rh}(111) \cdot{ }^{32,36-40} \mathrm{~A}$ strong preference for binding in the pores of $\mathrm{h}-\mathrm{BN} / \mathrm{Rh}(111)$ at room temperature was demonstrated for naphthalocyanine. ${ }^{32}$ Phthalocyanines also exhibited preferential adsorption in the pores, close to the pore edge ("wall" in between the pore and wire regions); the mobility of the molecules within the pores was significant already at $77 \mathrm{~K}$, leading to blurred scanning tunneling microscopy (STM) images. 38,39

In this paper, we report on the adsorption of azobenzene $\left(\mathrm{C}_{6} \mathrm{H}_{5} \mathrm{~N}=\mathrm{NC}_{6} \mathrm{H}_{5}\right)$, a prototypical molecular switch, ${ }^{41-44}$ which was not yet studied on h-BN prepared on metal single crystals. The main aim of the present study is to search for the preferential adsorption site, bond strength, neighbor interaction, and characteristic conformation of the molecule via experimental (HREELS, STM, TPD) and density functional theoretical (DFT) investigations.

Gas-phase electron diffraction measurements with data analysis using some structural constraints obtained from $a b$ initio calculations indicated that the energetically most stable structure of separated trans-azobenzene molecules is planar, with a $C_{2 h}$ symmetry. ${ }^{45}$ However, the torsional barrier for one phenyl ring around an $\mathrm{N}-\mathrm{C}$ bond was found to be rather small: $\sim 0.065 \mathrm{eV}^{45}$ Density functional theory (DFT) as well as second- and fourth-order Møller-Plesset perturbation theory (MP2 and MP4) calculations yielded typically planar $C_{2 h}$ structures but in some cases indicated tilted phenyl groups, depending also on the type of basis sets used. ${ }^{45-51}$ For example, the MP2 method with a $6-31+G^{*}$ basis resulted in a torsional angle of $19.5^{\circ}$ for the two phenyl rings around the $\mathrm{N}-\mathrm{C}$ bonds measured from the planar structure, but the energy barrier to the planarity of both phenyl rings was calculated to be small, $\sim 0.04 \mathrm{eV} .^{45}$ Recent electron diffraction studies and theoretical results on trans stilbene indicate that the slight tendency for nonplanarity obtained for azobenzene in a few calculations can be attributed to the deficiency of the $\mathrm{ab}$ initio level used. ${ }^{52}$ Since the great majority of calculations and the more recent electron diffraction results suggested planar geometry, as mentioned above, we assume $C_{2 h}$ geometry for the isolated trans-azobenzene.

\section{METHODS}

The experiments were carried out in two separate ultrahighvacuum (UHV) systems evacuated down to $5 \times 10^{-8} \mathrm{~Pa}$. The first one was equipped with a commercial scanning tunneling microscope (RT-STM, WA-Technology), a cylindrical mirror analyzer with a central electron gun (Staib-DESA-100) for Auger electron spectroscopy (AES), and a quadrupole mass spectrometer (Balzers-Prisma). Note that, in this chamber, AES and mass spectrometry (MS) were mainly applied to check the surface/ad-metal cleanliness and the gas-phase composition. The $\mathrm{Rh}(111)$ sample was mounted on a transferable sample cartridge, equipped with facilities for indirect heating of the crystal by thermal emission of a Wfilament heated ohmically. For temperature measurement, a chromel-alumel thermoelement ( $\mathrm{K}$ type) spot-welded to the side of the probe was applied. The acquisition of STM images was performed by an MK2-A810 SPM controller plus an MK3HV1 smart piezo driver (SOFT-dB) and open source GXSM Python 2.7 software. STM images of $512 \times 512$ pixels were recorded in the constant-current (cc) mode at a bias of -1.0 or $-2.0 \mathrm{~V}$ on the sample and at anneling current in the range $0.02-2 \mathrm{nA}$, using chemically etched $\mathrm{W}$ tips. The final conditioning of the tip was performed during the scans with voltage $(1-5 \mathrm{~V})$ and current $(1-5 \mathrm{nA})$ pulses. The images are shown in top-view representation where brighter areas correspond to higher $Z$ values. The $X-Y-Z$ calibration of the STM images was performed by measuring the characteristic morphological parameters of a clean $\mathrm{Rh}(111)$ surface where the lateral nearest neighboring atomic distance of 0.27 $\mathrm{nm}$ and the step height of $0.22 \mathrm{~nm}$ were considered.

The second UHV system was equipped with an LK EELS 3000 spectrometer for high-resolution electron energy loss spectroscopy (HREELS), a quadrupole mass spectrometer (Balzers-Prisma) for temperature-programmed desorption (TPD), and a cylindrical mirror analyzer for AES. In HREEL spectra, the intensity of the elastic peak was typically in the range of $10^{4}-10^{5}$ counts-per-second (cps) with a resolution of $20-40 \mathrm{~cm}^{-1}$ full width at half-maximum (FWHM). All reported spectra were recorded with a primary beam energy of $6.5 \mathrm{eV}$ and at an incident angle of $60^{\circ}$, with respect to the surface normal in the specular direction, unless stated otherwise. Spectra are displayed normalized to the elastic peak intensity, if not stated otherwise. The $\mathrm{Rh}(111)$ crystal was mounted on a tantalum wire, which was connected via a copper block to a liquid nitrogen reservoir. The sample was heated resistively from 100 to $1100 \mathrm{~K}$; its temperature was monitored by a chromel-alumel thermocouple spot-welded to the edge of the crystal and was controlled with a feedback circuit to provide a linear heating rate of ca. $4 \mathrm{~K} / \mathrm{s}$ for TPD measurements.

In both chambers, one-side-polished $\mathrm{Rh}(111)$ single crystals (dia. $6 \mathrm{~mm} \times 1.5 \mathrm{~mm}$, orientation accuracy: $0.1^{\circ}$ ) purchased from MaTeck Ltd. were used. The samples were routinely cleaned applying cycles of $\mathrm{Ar}^{+}$ion sputtering $\left(5-10 \mu \mathrm{A} / \mathrm{cm}^{2}\right.$, $1.5 \mathrm{keV}, 20 \mathrm{~min}$ ) at $300 \mathrm{~K}$ and $10 \mathrm{~min}$ annealing in UHV at $1100-1250 \mathrm{~K}$. The sample was oxidized in $3 \times 10^{-8} \mathrm{mbar}$ of $\mathrm{O}_{2}$ at $1000 \mathrm{~K}$ afterward, followed by annealing in UHV at $1100-1250 \mathrm{~K}$ for $5 \mathrm{~min}$. h-BN monolayers were prepared at $1000-1050 \mathrm{~K}$ via thermal decomposition of $>99.8 \%$ purity borazine, a product of Katchem Ltd. Adsorption of azobenzene was performed from the background $\left(1 \times 10^{-7} \mathrm{mbar}\right)$ controlled via a leak valve attached to the UHV chambers. The glass bulb containing azobenzene could be pumped separately before/during adsorption.

To obtain a deep theoretical insight into the adsorption properties of azobenzene on the $\mathrm{h}-\mathrm{BN} / \mathrm{Rh}(111)$ surface, DFT calculations are performed employing the Vienna $\mathrm{Ab}$ initio simulation package (VASP) $)^{53,54}$ with the projector augmented wave (PAW) method ${ }^{55}$ for the electron-ion interaction and with the optB86b-vdW functional ${ }^{56,57}$ for dispersion correction. Our supercell slab model consists of three Rh atomic layers surfaced in the (111) crystallographic orientation with $12 \times 12 \mathrm{Rh}$ atoms in each layer, followed by the h-BN overlayer in a $13 \times 13$ superstructure, corresponding to the lattice mismatch ${ }^{31}$ of h-BN and $\mathrm{Rh}(111)$ (the experimental inplane lattice constant of $\mathrm{Rh}$ is $2.689 \AA)$. Altogether, $770(3 \times$ $144(\mathrm{Rh})+2 \times 169(\mathrm{~B}+\mathrm{N}))$ atoms reside in the supercell, ${ }^{33}$ which serves as the substrate for the adsorption of azobenzene molecules. Including the molecules, a minimum-15- $\AA$-thick vacuum layer is added to the supercell in the perpendicular (111) direction to avoid unphysical interactions and to properly describe the vacuum tail of the electron wave functions for STM simulations.

The energetically preferred adsorption geometry of a single azobenzene molecule is determined from a total energy 
comparison of a set of 50 configurations after geometry optimizations without and with dipole correction, ${ }^{58}$ where the reported total energy differences correspond to the whole supercell. The initial configurations correspond to trans- or cisazobenzene, where their $\mathrm{N}$ or $\mathrm{N}$-nearest $\mathrm{C}$ atoms are above six surface adsorption sites (pore-N, pore-B, wall-N, wall-B, wire$\mathrm{N}$, wire- $\mathrm{B}$ ), all of these in two different orientations: rotated by $90^{\circ}$ with respect to each other. These combinations result in 48 configurations. Additionally, two more configurations are considered, where the $\mathrm{N}=\mathrm{N}$ double bond of a trans- or a cisazobenzene is above a $\mathrm{B}-\mathrm{N}$ bond in the pore region of $\mathrm{h}-\mathrm{BN} /$ $\mathrm{Rh}(111)$. After the energetically most favored adsorption geometry of a single azobenzene molecule is found, adsorption of a second molecule is considered in eight selected positions to obtain information about molecule-molecule interactions and possible molecular growth modes. During geometry optimizations, the $\Gamma$ point samples the Brillouin zone, the bottom $\mathrm{Rh}$ layer is fixed, and all other atoms are freely relaxed until the residual force acting on all individual atoms becomes smaller than $0.02 \mathrm{eV} / \AA \AA$. A $3 \times 3 \times 1 k$-point sampling is applied when calculating the adsorption energies, Bader charges, ${ }^{59-61}$ charge transfer, molecule-molecule interactions, and STM images. The reported STM images are calculated with an s-type tip (Tersoff-Hamann approximation) using the revised Chen method ${ }^{62}$ implemented in the BSKAN code. ${ }^{63,64}$

\section{RESULTS AND DISCUSSION}

3.1. Experimental Studies. 3.1.1. Temperature-Programmed Desorption of Azobenzene from $h-B N / R h(111)$. It is known from the literature that the multilayer of azobenzene desorbs at $\sim 240 \mathrm{~K}^{44}$ In this work, TPD measurements were conducted in the monolayer regime to understand whether the heterogeneity of the surface is reflected also in the desorption spectra. The masses $m / e=$ 51 and $m / e=77$ were followed, which gave an intense contribution to the mass spectrum of azobenzene. Based on

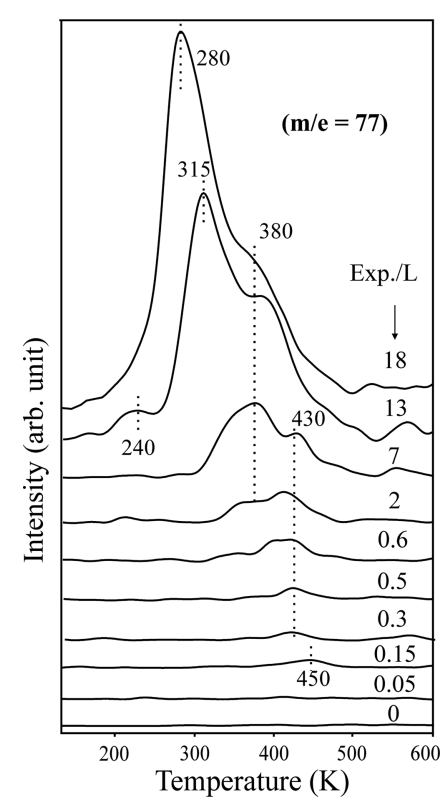

Figure 1. TPD spectra at $m / e=77$, assigned to azobenzene molecular desorption, after increasing exposures of azobenzene on h-BN/ $\mathrm{Rh}(111)$ at $130 \mathrm{~K}$. the results (Figure 1), the following conclusions can be drawn after exposure of azobenzene at $130 \mathrm{~K}$.

(i) At small exposures, the desorption maximum is at about $430-450 \mathrm{~K}$, which stepwise shifts down to $380 \mathrm{~K}$ up to $\sim 7 \mathrm{~L}$ exposures. These states correspond very likely to the adsorption in the energetically most stable pore adsorption sites. We will discuss the energetically favorable adsorption geometries later in the DFT Section in Section 3.2. Nevertheless, the variety of adsorption geometries in the pore region and at the borderline of pores and walls explains the relatively significant changes in the peak temperatures in this exposure regime, not to mention the possible effect of lateral molecule-molecule interactions.

(ii) By increasing the exposure of azobenzene, we can distinguish an additional desorption peak (at $315 \mathrm{~K}$ ) from the monolayer. Furthermore, it shifts down to even lower temperatures $(280 \mathrm{~K})$ and increases in intensity with exposure. These features are assigned to the population of energetically less favored adsorption sites in the wall and wire regions at larger exposures. In harmony with this observation, STM results indicate that these sites are typically uncovered at and above 320 $\mathrm{K}$, and even in the pores, there are still free adsorption centers (Figure 6). According to STM images and DFT models, the area of pores is comparable to the area of wire and wall regions. In light of these, it is not surprising that the TPD peak area increased by this extent $(18 \mathrm{~L})$ compared to the $380 \mathrm{~K}$ peak. Moreover, previous TPD studies of azobenzene adsorption on $\mathrm{Au}(111)$ and $\operatorname{Ag}(111)$ revealed that the onset of the desorption from the monolayer can substantially shift down to lower temperatures for dense layers, when approaching the saturation of the first layer, due to molecule-molecule interactions. ${ }^{44}$ Therefore, we cannot exclude a contribution of molecules in the pores to the main TPD peak at $280 \mathrm{~K}$ observed at $18 \mathrm{~L}$.

(iii) Since in this study we are only concerned with the monolayer adsorption of azobenzene, we do not present multilayer spectra. However, we can observe a sharp small peak at $240 \mathrm{~K}$ even at $13 \mathrm{~L}$ exposure. This peak does not increase after further exposure (at $18 \mathrm{~L}$ ), while the $280 \mathrm{~K}$ peak develops. One possible explanation for this phenomenon could be that we observe here desorption from the sample holder, but we assume an interpretation, which seems more likely. Namely, that after filling the pores with a single layer of azobenzene, at higher exposures, in parallel with stepwise formation of the monolayer in the wire regions, adsorption of azobenzene may occur on top of the first molecular layer in the pores as a minority process, on a "sticks where it hits" basis. This explains why we cannot observe a significantly larger contribution from the multilayer when the exposure increased from $13 \mathrm{~L}$ to $18 \mathrm{~L}$. Integrated TPD areas show a saturation of the monolayer only around $18 \mathrm{~L}$, at which exposure we reach presumably the full monolayer coverage.

TPD results demonstrate well the inhomogeneity of adsorption sites on the h-BN nanomesh, and with increasing exposure, desorption maximums shift toward lower temperatures. The trend is that the stability decreases on the order of pore-wall-wire regions of the $\mathrm{BN}$ monolayer as revealed by 
STM and DFT results presented below. Furthermore, the calculated adsorption energy for the trans molecule with the most stable geometry in the pore $(-1.49 \mathrm{eV})$, of course with an opposite sign, is rather close to the desorption activation energy $\left(E_{\mathrm{a}}=1.17 \mathrm{eV}\right)$ determined by a simple Redhead analysis of low-exposure TPD curves $\left(T_{\mathrm{p}}=450 \mathrm{~K}\right)$ assuming a preexponential factor of $10^{13} \mathrm{~s}^{-1}$. Although we are aware of the limitations of Redhead analysis compared to other more reliable methods, ${ }^{65}$ this approximation can also demonstrate the conformity of our calculated and experimental results. Schulze and co-workers ${ }^{44}$ applied the more reliable and accurate "complete analysis" method of TPD analysis for azobenzene adsorption energies on $\mathrm{Au}(111)$ single crystals. Even with that method, they obtained a significant difference between the experimental and calculated binding energy values on the $\mathrm{Au}(111)$ sample for azobenzene adsorption (1.00 \pm 0.15 vs $1.67 \mathrm{eV}$ ). We have discussed our results in detail in the Supporting Information.

3.1.2. High-Resolution Electron Energy Loss Experiments. The vibrational spectrum of the nanomesh itself (Figure 2

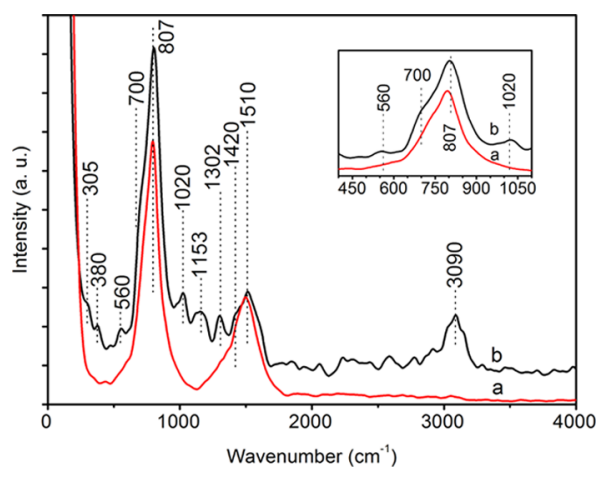

Figure 2. HREELS spectrum of (a) h-BN/Rh(111) and (b) h-BN/ $\mathrm{Rh}(111)$ after $100 \mathrm{~L}$ azobenzene exposure at $170 \mathrm{~K} .1 \mathrm{~L}=10^{-6}$ Torr $\times \mathrm{s}$, where 1 Torr $=1.33$ mbar.

spectrum a) consists of two strong peaks at 795 and $1510 \mathrm{~cm}^{-1}$ and of a shoulder at $\sim 1400 \mathrm{~cm}^{-1}$, which can be assigned to the out-of-plane transverse optical phonon mode $\left(\mathrm{TO}_{\perp}\right)$, the inplane longitudinal optical phonon mode (LO), and the inplane transverse optical phonon mode $\left(\mathrm{TO}_{\|}\right)$, respectively. The second harmonic of $\mathrm{TO}_{\perp}$ may also contribute to the $\mathrm{LO}$ peak. $^{66}$

The adsorption of an azobenzene multilayer at $170 \mathrm{~K}$ led to the appearance of new peaks (Figure 2 spectrum $b$ ), attributed to different vibrational modes of the molecule listed in Table 1. For comparison, IR data (both experimental and calculated) from the literature are also shown. Due to the $C_{2 h}$ symmetry of the molecule, $A_{u}$ and $B_{u}$ modes are IR active, while $A_{g}$ and $B_{g}$ modes are Raman active. Among the IR-active modes, $B_{u}$ vibrations are in-plane, while $A_{u}$ modes are out-of-plane. The more intense IR peaks are set in boldface. Note that all normal modes above $1000 \mathrm{~cm}^{-1}$ are in-plane, while the majority of vibrations below $1000 \mathrm{~cm}^{-1}$ are out-of-plane. We only observed modes of azobenzene and h-BN on our surface, indicating that the molecule adsorbed intact on the nanomesh. The adsorption of azobenzene on $\mathrm{h}-\mathrm{BN} / \mathrm{Rh}(111)$ led to the diminution of LO loss of the nitride at $1510 \mathrm{~cm}^{-1}$ due to the shadowing effect of the molecules. Since this peak overlaps with the $\delta(\mathrm{C}-\mathrm{H})$ mode of azobenzene at $\sim 1480 \mathrm{~cm}^{-1}$, it is difficult to identify the intensity originated from the nitride
Table 1. HREELS Losses Observed after Azobenzene Adsorption at $170 \mathrm{~K}$ and Their Assignment ${ }^{a}$

\begin{tabular}{|c|c|c|c|c|}
\hline $\begin{array}{c}\text { mode } \\
\text { description }^{51}\end{array}$ & symmetry & $\begin{array}{l}\text { IR spectrum in } \\
\text { Ar matrix } \\
\left(\mathrm{cm}^{-1}\right)^{51}\end{array}$ & $\begin{array}{l}\text { calculated } \\
\text { eigenfrequency } \\
\left(\mathrm{cm}^{-1}\right)^{51}\end{array}$ & $\begin{array}{c}\text { azobenzene on } \\
\text { h-BN/Rh(111)- } \\
\text { HREELS- } \\
\text { present study }\end{array}$ \\
\hline$\nu(\mathrm{C}-\mathrm{H})$ & $\mathrm{B}_{\mathrm{u}}$ & $\begin{array}{c}\text { 3104, 3093, } \\
\text { 3087, 3076, } \\
\text { 3070, 3057, } \\
3046,3015\end{array}$ & $\begin{array}{l}3143,3133 \\
3124,3114 \\
3104\end{array}$ & 3090 \\
\hline$\nu(\mathrm{C}-\mathrm{C})$ & $\mathrm{B}_{\mathrm{u}}$ & $\begin{array}{l}1595,1593 \\
1589,1588\end{array}$ & 1595,1578 & 1510 \\
\hline$\delta(\mathrm{C}-\mathrm{H})$ & $\mathrm{B}_{\mathrm{u}}$ & 1487,1457 & 1473,1446 & 1420 \\
\hline $\begin{array}{c}\nu(\mathrm{C}-\mathrm{C}) \\
\delta(\mathrm{C}-\mathrm{H})\end{array}$ & $\mathrm{B}_{\mathrm{u}}$ & 1307,1298 & 1353,1295 & 1302 \\
\hline$\nu(\mathrm{C}-\mathrm{N})$ & $\mathrm{B}_{\mathrm{u}}$ & 1221 & 1225 & \\
\hline$\delta(\mathrm{C}-\mathrm{H})$ & $\mathrm{B}_{\mathrm{u}}$ & $\begin{array}{c}1158,1152 \\
1148\end{array}$ & 1140 & 1153 \\
\hline$\delta(\mathrm{C}-\mathrm{H})$ & $\mathrm{B}_{\mathrm{u}}$ & 1072 & 1073 & \\
\hline$\nu(\mathrm{C}-\mathrm{C})$ & $\mathrm{B}_{\mathrm{u}}$ & 1024,1019 & 1017 & 1020 \\
\hline$\gamma(\mathrm{C}-\mathrm{H})$ & $A_{u}$ & 927,925 & 928 & 950 \\
\hline$\gamma(\mathrm{C}-\mathrm{H})$ & $A_{u}$ & 781,778 & 780 & 807 \\
\hline$\tau(\mathrm{C}-\mathrm{C})$ & $A_{u}$ & 689 & 685 & 700 \\
\hline$\gamma(\mathrm{N}-\mathrm{C})$ & $A_{u}$ & $550,547,546$ & 541 & 560 \\
\hline $\begin{array}{l}\delta(\mathrm{C}-\mathrm{C}) \\
\delta(\mathrm{N}-\mathrm{C}- \\
\mathrm{C})\end{array}$ & $\mathrm{B}_{\mathrm{u}}$ & 536,521 & 532,514 & \\
\hline$\tau(\mathrm{C}-\mathrm{C})$ & $A_{u}$ & & 403,295 & 380 \\
\hline
\end{tabular}

${ }^{a}$ For comparison, calculated and experimental IR peak positions of trans-azobenzene are also displayed. The IR peaks observed with strong or medium intensity are listed in boldface.

alone. Similarly, the most intense bands of the IR spectrum, $\gamma(\mathrm{C}-\mathrm{H})$ at $780 \mathrm{~cm}^{-1}$ and $\tau(\mathrm{C}-\mathrm{C})$ at $690 \mathrm{~cm}^{-1}$, overlap with the $\mathrm{TO}_{\perp}$ nitride loss $\left(795 \mathrm{~cm}^{-1}\right)$. In the HREELS spectrum, a slight intensity increase was observed at $807 \mathrm{~cm}^{-1}$ upon azobenzene adsorption (Figure 2), implying that the attenuation of the $\mathrm{TO}_{\perp}$ nitride mode due to the shadowing effect of azobenzene is overcompensated by the appearance of the strong out-of-plane $\gamma(\mathrm{C}-\mathrm{H})$ and $\tau(\mathrm{C}-\mathrm{C})$ modes. The presence of azobenzene on the surface also led to the broadening of the $\sim 800 \mathrm{~cm}^{-1}$ peak toward smaller wavenumbers and the appearance of a shoulder at $\sim 700 \mathrm{~cm}^{-1}$, attributed to the $\tau(\mathrm{C}-\mathrm{C})$ mode (Figure 2 inset).

In the next experiment, azobenzene was dosed at 300-310 $\mathrm{K}$, to avoid the growth of a multilayer. The obtained HREELS spectra are shown in Figure 3. Note that the sticking coefficient

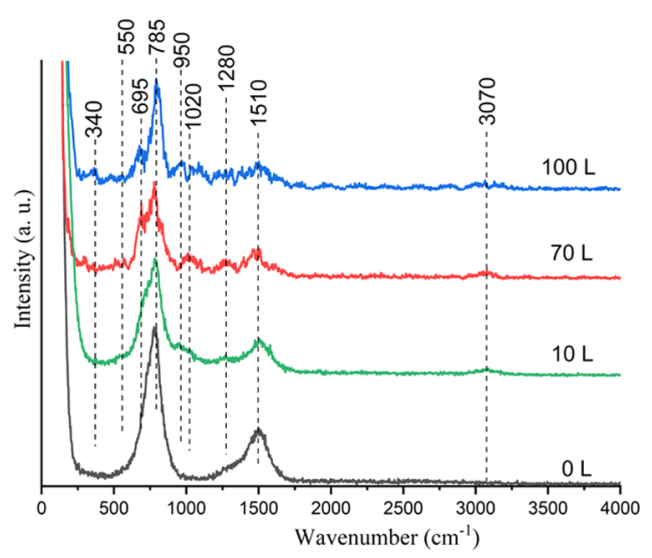

Figure 3. HREELS spectrum after increasing exposure of h-BN/ $\mathrm{Rh}(111)$ to azobenzene at $300 \mathrm{~K}$. 
can be smaller in this case due to the higher substrate temperature compared to the TPD measurements presented above. Losses characteristic of azobenzene appeared with stepwise increasing intensity. At the same time, the h-BN phonon modes gradually attenuated due to the shadowing effect of the increasing number of molecules at higher exposures. This phenomenon resulted in a more complex (sometimes nonmonotonous) behavior of the azobenzene losses overlapping with the $\mathrm{h}-\mathrm{BN}$ peaks. It is interesting to note that the $\nu\left(\mathrm{C}-\mathrm{H}\right.$ ) peak at $3070 \mathrm{~cm}^{-1}$ (which is one of the most intense peaks with FT-IR spectroscopy) was very weak, indicating that the $\mathrm{C}-\mathrm{H}$ bonds are approximately parallel to the surface. It suggests that the trans-azobenzene molecule adsorbs (nearly) parallel to the nitride monolayer. Although the in-plane modes between 1000 and $1600 \mathrm{~cm}^{-1}$ are visible, the impact scattering mechanism can also contribute to their appearance.

To corroborate the hint for the adsorption geometry, angleresolved HREELS measurements were also performed. It is known that the dipole scattering mechanism is only operational at incident and detection angles near the specular geometry, while the cross section of the impact scattering mechanism typically changes less abruptly as a function of the detection angles. ${ }^{67}$ Therefore, a steep intensity loss is observed for peaks dominated by the dipole mechanism, when departing from the specular geometry. Note that due to the presence of the metal substrate ("mirror charges") only normal modes having a dynamic dipole component perpendicular to the surface can be excited by dipole scattering.

First, HREELS spectra of the nanomesh itself obtained at different angles of incidence are shown in Figure 4. A steep

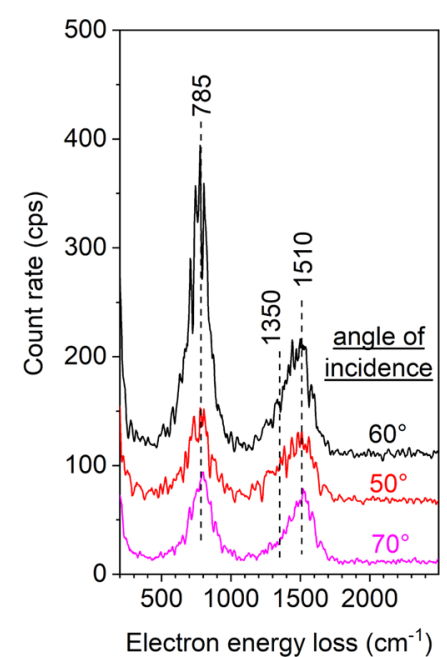

Figure 4. HREELS spectra of $\mathrm{h}-\mathrm{BN} / \mathrm{Rh}(111)$ obtained at different electron incidence angles. The specular geometry is at $60^{\circ}$. Spectra are not normalized to the elastic peak intensity.

intensity loss is observed for the out-of-plane $\mathrm{TO}_{\perp}$ mode (785 $\left.\mathrm{cm}^{-1}\right)$ at the off-specular geometries: its intensity at 50 and $70^{\circ}$ was only $30 \%$ of the specular intensity. On the other hand, a much milder angular dependence was found for the in-plane LO mode $\left(1510 \mathrm{~cm}^{-1}\right)$ : its intensities at 50 and $70^{\circ}$ were 50 and $65 \%$ of the specular intensity, respectively. This is reasonable considering that the h-BN "plane" is nearly parallel to the $\mathrm{Rh}(111)$ surface. The undulating character of the nanomesh may result in some dipole activity of the in-plane modes of h-BN as well, but the corrugation of the nanomesh $(\sim 0.2 \mathrm{~nm})$ is relatively small compared to the periodicity of the superstructure $(\sim 3.2 \mathrm{~nm})$.

Next, HREELS spectra were collected at different angles of incidence after exposing $\mathrm{h}-\mathrm{BN} / \mathrm{Rh}(111)$ to $100 \mathrm{~L}$ of azobenzene at $300 \mathrm{~K}$ (Figure 5). Obviously, the out-of-plane

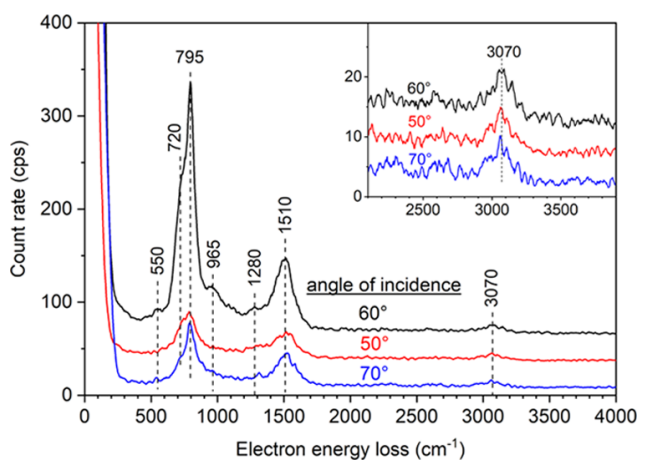

Figure 5. HREELS spectra obtained at different angles of incidence after the exposure of h-BN/Rh(111) to $100 \mathrm{~L}$ of azobenzene at $300 \mathrm{~K}$. The specular geometry is at $60^{\circ}$. Spectra are not normalized to the elastic peak intensity. The $\mathrm{C}-\mathrm{H}$ stretch region is shown magnified in the inset.

modes $\left(720,796,965 \mathrm{~cm}^{-1}\right)$ suffered a strong intensity loss at the off-specular geometries, while the attenuation of the inplane peaks $\left(1280,1510 \mathrm{~cm}^{-1}\right)$ was milder. The $\mathrm{C}-\mathrm{H}$ stretch peak $\left(3070 \mathrm{~cm}^{-1}\right)$ is particularly meaningful in this sense because h-BN has no contribution to the spectra in this region. The $\mathrm{C}-\mathrm{H}$ stretch intensity is almost invariant to the applied measurement geometries, strongly indicating that the $\mathrm{C}-\mathrm{H}$ bonds are essentially parallel to the surface. The change in the molecular dipole during a $\mathrm{C}-\mathrm{H}$ stretch (dynamic dipole) is parallel to the $\mathrm{C}-\mathrm{H}$ bond itself. $\mathrm{C}-\mathrm{H}$ stretch motions are coupled together in various $\mathrm{C}-\mathrm{H}$ stretch normal modes, but all are certainly characterized by in-plane dynamic dipoles. In case a phenyl ring were tilted with respect to the surface, at least some of the $\mathrm{C}-\mathrm{H}$ stretch modes should be dipole-active, which was not observed. Therefore, the presented angleresolved measurements confirm that the adsorption geometry of azobenzene is predominantly parallel to the h-BN monolayer. If both phenyl rings are parallel to the surface, then it also implies that the molecule is in its trans conformation.

3.1.3. Scanning Tunneling Microscopy Measurements. Here, we present several characteristic STM images taken on the h-BN/ $\mathrm{Rh}(111)$ surfaces exposed to azobenzene at $320 \mathrm{~K}$. The hexagonal nanomesh structure before the gas exposure can exhibit basically two different contrasts: honeycomb and disk shape (Figure 6A, top and bottom half). In the case of the honeycomb structure, the wire regions are bright and the pore regions are dark. This contrast is reversed for the disk-shaped structure where the pore regions are bright. This type of double appearance of the STM images taken on the h-BN monolayer supported by metal single crystals has been described in details over the last few years and associated with the change of d-band-dependent tunneling probability. 7,31 It is important to note, however, that the spontaneous relay between the two imaging appearances suggests that this feature is not simply a bias-dependent change, but it is certainly based on the sudden chemical change of the tip termination itself. 


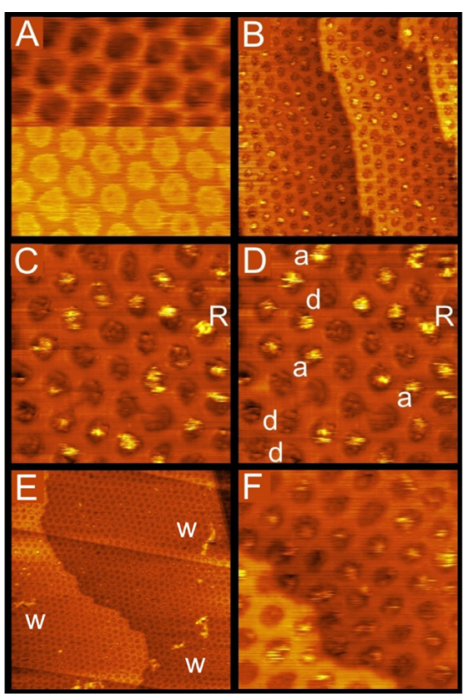

Figure 6. STM images recorded at room temperature (A) before and after different exposures of azobenzene onto the $\mathrm{h}-\mathrm{BN} / \mathrm{Rh}(111)$ surface at $320 \mathrm{~K}$ : (B-D) $30 \mathrm{~L}$ and (E, F) $90 \mathrm{~L}$. Size of the images: (A, C, D, F) $20 \times 20 \mathrm{~nm}^{2}$, (B) $50 \times 50 \mathrm{~nm}^{2}$, and (E) $100 \times 100 \mathrm{~nm}^{2}$. Parameters found for the best imaging of the surface covered by azobenzene molecules: $U_{\mathrm{t}}=-1 \mathrm{~V}$ voltage on the sample and $I_{\mathrm{t}}=20$ pA current.

To detect individual molecules adsorbed at $320 \mathrm{~K}$, the substrate was exposed to $30 \mathrm{~L}$ of $\left(\mathrm{C}_{6} \mathrm{H}_{5} \mathrm{~N}\right)_{2}$. The constantcurrent STM images recorded in two different scales are shown in Figure 6B,C. The image (B) of $50 \times 50 \mathrm{~nm}^{2}$ exhibits clearly the appearance of nanodots distributed more or less uniformly on the atomic terraces of the $\mathrm{Rh}(111)$ surface covered by the h-BN nanomesh without any preference at the step lines. However, the dots assigned certainly to the individual azobenzene molecules adsorb exclusively in the pore regions as they are even more unambiguously discernible on the image (C) of $20 \times 20 \mathrm{~nm}^{2}$. The height of these features is approximately $0.15 \mathrm{~nm}$, and they fill approximately half of the pore regions with a diameter of $2 \mathrm{~nm}$. A more precise determination of the morphology of the molecules is very difficult due to the high mobility activated also by the tip itself at this temperature (RT). To demonstrate this effect, let us observe two images recorded subsequently after each other in nearly the same lateral positions (Figure 6C,D). On both images of $20 \times 20 \mathrm{~nm}^{2}$, a reference dot is marked by " $\mathrm{R}$ " on the right side of the region. Comparing the other dots in the two records, it is clear that the molecules marked "a" appeared as new ones in the image (D) and they were not present in the corresponding "pore"-s in the first record (C). At the same time, the molecules present in the first record disappeared from the pores marked by "d". From the fact that the number of molecules that appeared and disappeared is nearly the same, it can be concluded that the tunneling tip activates intensively the surface diffusion (jump from a pore site to another pore site) of the adsorbed molecules without activating their desorption. Naturally, an increase of the tunneling current may activate a desorption event, as well. We have found the best parameters for a relatively "calm" imaging: $-1 \mathrm{~V}$ on the sample with a tunneling current of $20 \mathrm{pA}$. It is also observable in images (C) and (D) that some dots are brighter and exhibit a more extended shape laterally (in some cases a double-dot structure), which can be caused by a doubled adsorption in a pore site or a doubled imaging of the same individual molecule.
Naturally, concerning the shape of the adsorbed azobenzene molecules, imaging at much lower temperatures would provide a more detailed result.

At a higher exposure of $90 \mathrm{~L}$ of azobenzene, two different arrangements of the new features were observed (Figure 6E,F): (i) the larger scale image of $100 \times 100 \mathrm{~nm}^{2}$ exhibits elongated wormlike islands (accumulated 1D molecular stripes) of 1-2 $\mathrm{nm}$ width as marked by "w" letters in the image (E); and (ii) individual nanodots as described above fitting to the lateral distribution of the pore regions (F). The former morphology suggests an island formation of the azobenzene molecules due to a short-range attractive interaction between them. A possible role of line defects of the nanomesh in the formation of the wormlike structures cannot be excluded completely. These islands occupy naturally also the wire regions where the bond is weaker to the nanomesh. At the same time, the latter (ii) morphology clearly refers to the template effect of the pore regions.

3.2. DFT Calculations. First, the energetically preferred adsorption geometry of a single azobenzene molecule is determined on the $\mathrm{h}-\mathrm{BN} / \mathrm{Rh}(111)$ nanomesh structure (the surface unit cell is shown in the top row of Figure 7 ). We are interested in which isomer of the azobenzene (trans or cis) is preferentially bound to the surface and whether there is a preferred surface region (pore, wall, wire; see the top row of Figure 7) for adsorption. After geometry optimizations of the considered 50 adsorption structures (for a description, see

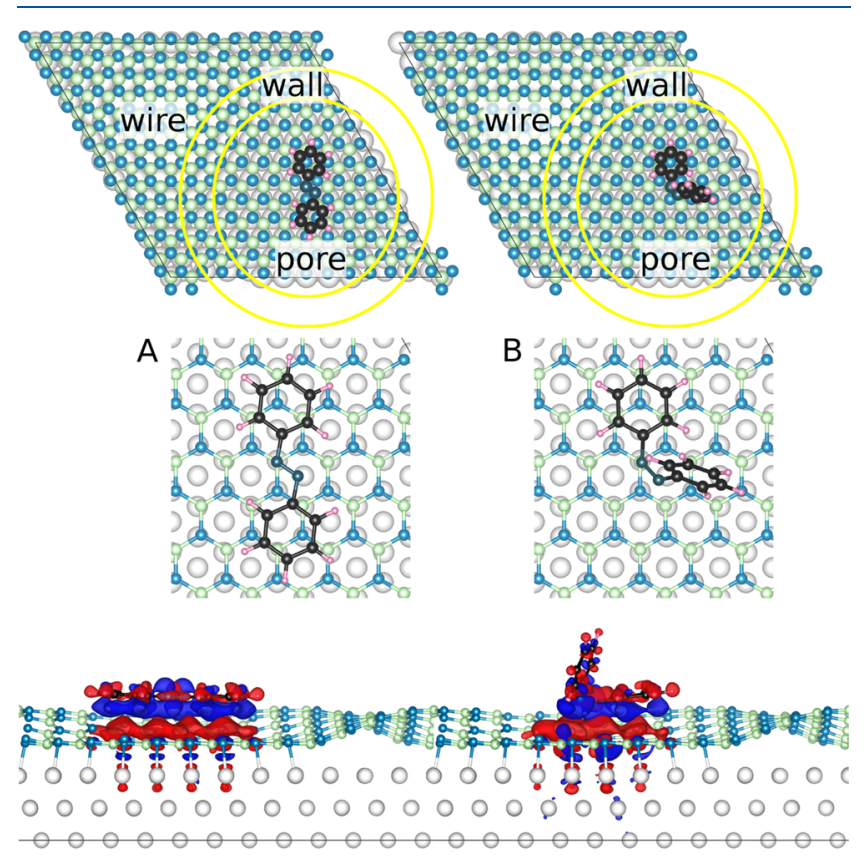

Figure 7. Energetically favored trans- (A, left) and cis- (B, right, 0.82 $\mathrm{eV}$ higher in total energy) azobenzene adsorption configurations after geometry optimizations with dipole correction (top: top view showing the surface unit cell and the different surface regions: pore, wall, wire; middle: top view zoomed on the molecules; bottom: side view showing the charge transfer upon adsorption). For both isomers, the pore region of $\mathrm{h}-\mathrm{BN} / \mathrm{Rh}(111)$ is preferred (see also Table 2). The 3D charge transfers $\left(\rho_{\text {sub }+ \text { mol }}-\rho_{\text {sub }}-\rho_{\text {mol }}\right)$ upon molecular adsorption on the substrate are explicitly shown at the bottom of the figure (isosurface value: $2 \times 10^{-4} \mathrm{lel} / \AA^{3}$; blue, electron accumulation; red, electron depletion). Colors for the atoms: gray ( $\mathrm{Rh})$, light green (B), light blue (substrate-N), dark blue (molecule-N), black (C), pink (H). 
Section 2), the energetically most stable adsorption configuration was a trans molecule in the pore, shown in Figure 7A. The total energy ranges of the relaxed configurations in the characteristic surface areas (pore, wall, wire) with respect to this identified overall energy minimum are shown in Table 2.

Table 2. Relative Total Energy Ranges for the Considered Single Azobenzene Molecular Adsorption Configurations on Various Surface Regions of h-BN/Rh(111) with Respect to the Identified Overall Energy Minimum $(0 \mathrm{eV})^{a}$

$\begin{array}{cc} & \text { relative total energy ranges }(\mathrm{eV}) \\ \text { trans-azobenzene } & \\ \text { pore ( } 9 \text { configurations): } & 0.00-0.08 \\ \text { wall ( } 8 \text { configurations): } & 0.04-0.27 \\ \text { wire ( } 8 \text { configurations): } & 0.33-0.45 \\ \text { cis-azobenzene } & \\ \text { pore ( } 9 \text { configurations): } & 0.82-0.91(0.00-0.09) \\ \text { wall ( } 8 \text { configurations): } & 0.88-1.14(0.06-0.32) \\ \text { wire ( } 8 \text { configurations): } & 1.17-1.25(0.35-0.43)\end{array}$

${ }^{a}$ For the cis-azobenzene, the relative total energies are also reported (in parenthesis) with respect to the energetically favored cisazobenzene (in the pore; see Figure $7 \mathrm{~B}$ ), which is $0.82 \mathrm{eV}$ higher in total energy than the overall favored trans-azobenzene in the pore region of the nanomesh (denoted $0 \mathrm{eV}$; see Figure $7 \mathrm{~A}$ ).

As can be seen, these relative total energy ranges are quite narrow $(\leq 0.09 \mathrm{eV}$ for pore $(0.08 \mathrm{eV}$ for trans and $0.09 \mathrm{eV}$ for cis), $\leq 0.26 \mathrm{eV}$ for wall ( $0.23 \mathrm{eV}$ for trans and $0.26 \mathrm{eV}$ for cis), and $\leq 0.12 \mathrm{eV}$ for wire $(0.12 \mathrm{eV}$ for trans and $0.08 \mathrm{eV}$ for cis $)$ ) and they are practically distinct in the different surface regions, with a very small energetic overlap between pore and wall regions. The preferred surface area for the adsorption of both azobenzene isomers is clearly the pore region, followed by the wall region, and the nanomesh wire region is unfavored. These results are in agreement with the concluded heterogeneity of adsorption sites based on Figure 1, and they are in stark contrast to that of ethanol adsorption ${ }^{68}$ on h-BN/Rh(111), where no such preference for adsorption in the pore region of the nanomesh has been found. Furthermore, it is obtained that the best trans-azobenzene adsorption configuration is $0.82 \mathrm{eV}$ lower in total energy than the best cis-azobenzene. This clearly indicates a preference for trans-azobenzene adsorption on $h$ $\mathrm{BN} / \mathrm{Rh}(111)$. Note that the obtained trans-cis-azobenzene total energy difference of $0.82 \mathrm{eV}$ in favor of the trans isomer is very close to that of a similar study on the $\mathrm{MoS}_{2}$ surface, where $0.81 \mathrm{eV}$ was found. ${ }^{69}$ For the trans-cis energy difference in the gas phase, the value of $0.51 \mathrm{eV}$ is reproduced, ${ }^{69}$ and the trans isomer is favored due to the $\mathrm{N}=\mathrm{N}$ double bond, which enhances the delocalization of electrons in the $\pi$-conjugated flat molecule.

Figure 7 shows the most favored trans- (Figure 7A) and cisazobenzene (Figure 7B) adsorption configurations both located in the pore of the $\mathrm{h}-\mathrm{BN} / \mathrm{Rh}(111)$ nanomesh. Both aromatic rings of trans-azobenzene are found to be parallel to the surface. This is in agreement with the experimental conclusions in Section 3.1. One aromatic ring of cisazobenzene is also found to be close to parallel with the surface. All of these results are in agreement with those on $\mathrm{MoS}_{2}{ }^{69}$ For both isomers, a molecular $\mathrm{N}$ atom is preferred to be above a surface $\mathrm{B}$ atom, with such $\mathrm{B}-\mathrm{N}$ center-to-center distances of 3.11 and $2.60 \AA$ for the trans- and cis-azobenzene, respectively. These $\mathrm{B}-\mathrm{N}$ distance values are slightly lower, most likely due to the presence of $\mathrm{Rh}$ underneath $\mathrm{h}-\mathrm{BN}$, but show the same tendency as found for azobenzene monolayers on a graphene layer, where 3.30 and $2.95 \AA$ were, respectively, reported for trans and cis isomers. ${ }^{70}$

For our considered substrate, the adsorption energies $\left(E_{\mathrm{ads}}=\right.$ $\left.E_{\text {sub }+ \text { mol }}-E_{\text {sub }}-E_{\text {mol }}\right)$ are $-1.51 \mathrm{eV}$ (trans) and $-1.22 \mathrm{eV}$ (cis) without dipole correction, and these values slightly change to $-1.49 \mathrm{eV}$ (trans) and $-1.19 \mathrm{eV}$ (cis) with dipole correction taken into account. From chemical intuition, it seems straightforward that on inert surfaces, where vdW interactions dominate, the cis-azobenzene is less bound to the surface by having only one phenyl ring parallel to the substrate and the second phenyl ring is flipped up, in comparison to the trans-azobenzene, where both phenyl rings are parallel to the surface; therefore, this latter isomer binds stronger. This consideration is supported by our calculated detailed energetic data taking the $\mathrm{N}_{2}$ and the phenyl parts of the molecules separately, and the results are reported in Section S1 of the Supporting Information. Thus, the trans-azobenzene binds stronger to the $\mathrm{h}-\mathrm{BN} / \mathrm{Rh}(111)$ substrate than the cisazobenzene, and the difference between the adsorption energies is $0.29(0.30) \mathrm{eV}$ without (with) dipole correction. Interestingly, these values also match very well with the adsorption energy difference of $0.30 \mathrm{eV}$ between trans- and cisazobenzene adsorbed on $\mathrm{MoS}_{2}$, even though the adsorption energies themselves are smaller in that case: $-0.98 \mathrm{eV}$ (trans) and $-0.68 \mathrm{eV}$ (cis). ${ }^{69}$ This, together with the very close values of the total energy difference between trans- and cisazobenzene $(0.82$ and $0.81 \mathrm{eV})$, suggests that the h-BN/ $\mathrm{Rh}(111)$ and $\mathrm{MoS}_{2}$ substrates behave very similarly in terms of energetics concerning the adsorption difference between single trans- and cis-azobenzene. Of course, at larger quantities of azobenzene molecules, substantial differences are expected due to the heterogeneity of the h-BN nanomesh structure and the much different adsorption energy values. On less inert surfaces, like on $\mathrm{Cu}(100)$, the cis conformer of an azobenzene derivative is more stable than the trans because of stronger metal-N bonding. $^{71}$

The adsorbed molecules in Figure 7 are characterized by the following element-specific effective Bader charges $\left(Z_{\text {valence }}-\right.$ $Q_{\text {Bader }}$ ) averaged to one atom without (with) dipole correction: trans-N: $-0.50(-0.49)$ lel; trans-C: $-0.04(+0.02)$ lel; trans$\mathrm{H}:+0.15(+0.06)$ lel; cis- $\mathrm{N}:-0.46(-0.44)$ lel; cis-C: -0.06 $(-0.01)$ lel; cis-H: $+0.16(+0.09)$ lel. The obtained effective

Table 3. Element-Specific Bader Charge Differences $\left(Q_{\mathrm{sub}+\mathrm{mol}}-Q_{\mathrm{sub}}-Q_{\mathrm{mol}}\right)$ in lel Units upon the Adsorption of Trans- and Cis-Azobenzene Molecules (Denoted "mol") in Their Energetically Preferred Configurations (Shown in Figure 7) in the Pore of the h-BN/Rh(111) Substrate (Denoted "sub") ${ }^{a}$

$\begin{array}{lcccccccccc} & \text { sub-Rh } & \text { sub-B } & \text { sub-N } & \text { mol-N } & \text { mol-C } & \text { mol-H } & \text { mol-N2 } & \text { mol-phenyl1 } & \text { mol-phenyl2 } \\ \text { trans-azobenzene } & -0.037 & -0.011 & -0.054 & 0.041 & 0.066 & -0.005 & 0.041 & 0.029 & 0.032 \\ \text { cis-azobenzene } & -0.013 & -0.033 & -0.054 & 0.075 & 0.029 & -0.004 & 0.075 & 0.021 & 0.004\end{array}$

${ }^{a_{T}}$ The corresponding Bader charge differences for another partitioning of the molecules are also shown for N2 and the two phenyl rings separately. 


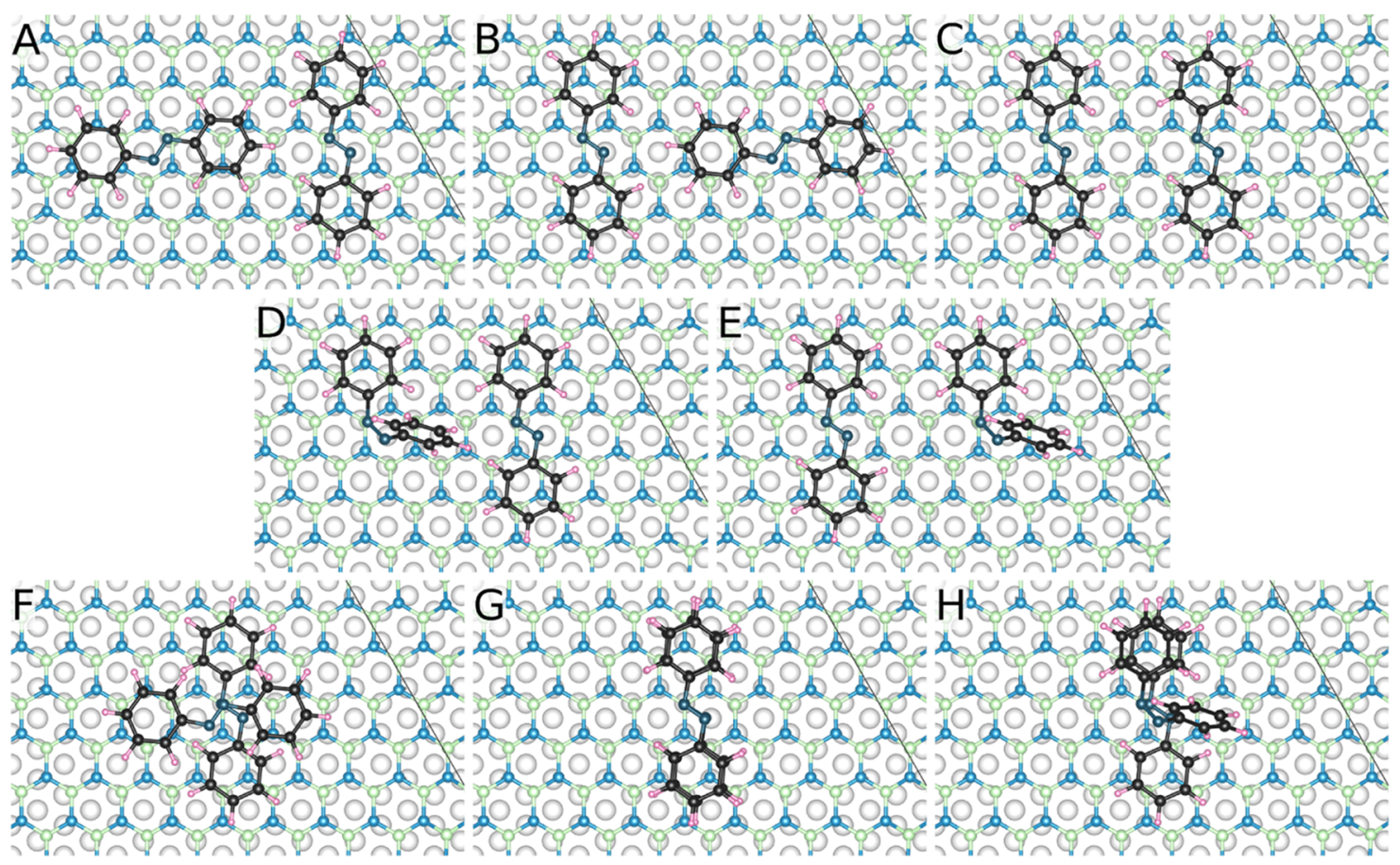

Figure 8. Energetic preference order of eight considered configurations for the adsorption of two azobenzene molecules in the pore region of h$\mathrm{BN} / \mathrm{Rh}(111)$, and their relative total energies with respect to that of the lowest one (trans(rot)-trans) without/with dipole correction: (A) trans $($ rot $)-\operatorname{trans}(0 / 0 \mathrm{meV}),(\mathrm{B})$ trans-trans $(\mathrm{rot})(3 / 4 \mathrm{meV}),(\mathrm{C})$ trans-trans $(52 / 54 \mathrm{meV}),(\mathrm{D})$ cis-trans $(788 / 815 \mathrm{meV}),(\mathrm{E})$ trans-cis $(882 /$ $913 \mathrm{meV}),(\mathrm{F})$ trans(rot)-above-trans $(953 / 967 \mathrm{meV}),(\mathrm{G})$ trans-above-trans $(1131 / 1136 \mathrm{meV})$, and $(\mathrm{H})$ cis-above-trans $(1595 / 1638 \mathrm{meV})$. The color schemes for the atoms are reported in the caption of Figure 7.

Bader charges on the $\mathrm{N}$ atoms of the molecules are larger in absolute value compared to those on $\mathrm{MoS}_{2},-0.28$ lel (trans) and -0.24 lel (cis), ${ }^{69}$ and this indicates a stronger bonding to $\mathrm{h}-\mathrm{BN} / \mathrm{Rh}(111)$ as discussed above comparing the adsorption energies. Detailed data on the Bader charges of the constituting atoms of the single azobenzene molecules shown in Figure 7 are reported in section S2 of the Supporting Information.

Figure 7 also shows the 3D charge transfers upon molecular adsorption $\left(\rho_{\text {sub }+ \text { mol }}-\rho_{\text {sub }}-\rho_{\text {mol }}\right)$. The rearrangement of the electrons to form the bonds between the molecules and the substrate is evident. The charge-transfer mechanism upon molecular adsorption is quantified in terms of calculating the element-specific Bader charge differences $\left(Q_{\text {sub+mol }}-Q_{\text {sub }}-\right.$ $Q_{\text {mol }}$ ), which are reported in Table 3 . While the substrate-N atoms donate an equal amount of partial electron charge to the molecule $(-0.054$ lel) for both trans- and cis-azobenzene, the charge transfer from the substrate- $\mathrm{B}$ and $\mathrm{Rh}$ atoms is rather different. More charge is transferred from the $\mathrm{Rh}$ for the trans $(-0.037 \mathrm{lel})$ than for the cis $(-0.013$ lel $)$ and concomitantly less charge is transferred from the B for the trans $(-0.011$ lel) than for the cis $(-0.033$ lel). At the molecular side, the charge transfer from $\mathrm{H}$ is very small $(<\mid 0.005 \mathrm{el})$, and all donated charge is received by the molecular $\mathrm{N}$ and $\mathrm{C}$ atoms, however, in different proportions. While 38\% $(0.041 /(0.041+0.066))$ of the received charge transfer is obtained by the molecular $\mathrm{N}$ atoms and $62 \%$ by the molecular $\mathrm{C}$ atoms for the trans, these values are $72 \%(0.075 /(0.075+0.029))$ by $\mathrm{N}$ and $28 \%$ by $\mathrm{C}$ for the cis. This difference, together with the enhanced charge transfer from the substrate- $\mathrm{B}$ atoms, indicates a dominating (substrate-B)-(molecule-N) bonding character for the cisazobenzene, which correlates well with the reduced (substrateB)-(molecule-N) center-to-center distance of $2.60 \AA$ in comparison to that of the trans-azobenzene, $3.11 \AA$. Table 3 also reports corresponding Bader charge differences for taking another partitioning of the molecule: $\mathrm{N}_{2}$ and two phenyl rings. While $\mathrm{N}_{2}$ has considerably less charge transfer for the trans $(0.041 \mathrm{lel})$ than for the cis $(0.075 \mathrm{lel})$, the phenyl rings have much more: trans, 0.029 and 0.032 lel (both parallel to the surface) and cis, 0.021 lel (almost parallel to the surface) and 0.004 lel (flipped). This difference, together with the proportionally more charge transfer to the molecular $\mathrm{C}$ atoms and the enhanced charge transfer from the substrate $\mathrm{Rh}$ atoms, indicates a dominating substrate-phenyl interaction for the trans-azobenzene. These findings explain the different adsorption energies of the two azobenzene isomers on the $h$ $\mathrm{BN} / \mathrm{Rh}(111)$ substrate.

By calculating the adsorption energies of representative single trans-azobenzene molecules in the wall and wire regions as well, we reproduced the experimentally found decreasing stability order of pore-wall-wire: $E_{\mathrm{ads}}=-1.49 \mathrm{eV}$ (pore), $-1.40 \mathrm{eV}$ (wall), $-1.17 \mathrm{eV}$ (wire), which is clear evidence for the template effect of the $\mathrm{h}-\mathrm{BN}$ nanomesh on $\mathrm{Rh}(111)$. For more details, the reader is referred to Section S6 of the Supporting Information.

Motivated by the possibility that a second molecular layer starts to grow before reaching the monolayer and by the presence of narrow molecular islands in Figure 6E, as the next step, in addition to the energetically most favored transazobenzene molecule in the pore of $\mathrm{h}-\mathrm{BN} / \mathrm{Rh}(111)$ (Figure 7), the adsorption of a second molecule is considered in eight selected positions to obtain information about possible molecular growth modes and molecule-molecule interactions. The relaxed geometries are shown in Figure 8, and their relative total energies calculated with and without dipole correction are reported in the figure caption. The configurations in Figure 8 are grouped as follows. The second trans- 
azobenzene molecule is adsorbed in the nanomesh pore beside the first one in different orientations and sequences so that the (substrate-B) $-($ trans-molecule2-N) bond is assured (top row: Figure $8 \mathrm{~A}-\mathrm{C})$; a cis-azobenzene molecule is adsorbed in the nanomesh pore beside the trans-azobenzene in two sequences so that the (substrate-B) $-($ cis-molecule- $\mathrm{N})$ bond is assured (middle row: Figure 8D,E); trans- or cis-azobenzene molecules are adsorbed above a trans-azobenzene in the nanomesh pore (bottom row: Figure $8 \mathrm{~F}-\mathrm{H}$ ). We find that this grouping naturally follows the energetic preference order of the adsorption configurations. The energetically preferred structures are two trans-azobenzenes beside each other in the pore of $\mathrm{h}-\mathrm{BN} / \mathrm{Rh}(111)$, and a composition of a rotated molecule by $90^{\circ}$ beside the other one is favored by about $50 \mathrm{meV}$ over parallel molecules. The best configuration is denoted "trans(rot)-trans", and the oppositely ordered composition, denoted trans-trans(rot), is practically degenerate in total energy. Note that two trans-azobenzenes along their long directions do not fit to the pore of the nanomesh (Figure 7A). The adsorption of a cis- and a trans-azobenzene in the pore is energetically much less favored. The obtained total energy differences of 788 (815) and 882 (913) $\mathrm{meV}$ without (with) dipole correction between cis-trans compared to trans-trans adsorptions are close to the difference between the single cisand trans-molecules, $0.82 \mathrm{eV}$ (Table 2). The observed variations are related to molecule-molecule interactions, where the cis-trans sequence is preferred over the trans-cis one (Figure $8 \mathrm{D}, \mathrm{E}$ ). The azobenzene molecular adsorption above a single trans-azobenzene is unfavored, as indicated by the relative total energies of the configurations (respectively, 953 (967) $\mathrm{meV}, 1131$ (1136) $\mathrm{meV}$, and 1595 (1638) $\mathrm{meV}$ without (with) dipole correction) shown in Figure 8F-H. This finding is in line with the experimentally derived conclusion on the stability of only the first molecular layer at room temperature, which is based on Figure 1.

The energetically preferred trans(rot)-trans configuration shown in Figure 8A is described further. The (substrate- $\mathrm{B}$ )(molecule-N) center-to-center distances are 3.12 and $3.29 \AA$ for the first trans- (on the right side of Figure 8A, denoted " $\mathrm{T}$ ") and the second (rotated) (on the left side of Figure 8A, denoted "TR") trans-azobenzene molecule, respectively. The adsorption energy of the coupled molecules is $E_{\text {sub+T+TR }}-E_{\text {sub }}$ $-E_{\mathrm{T}+\mathrm{TR}}=-3.05(-3.04) \mathrm{eV}$ without (with) dipole correction (here, sub denotes the $\mathrm{h}-\mathrm{BN} / \mathrm{Rh}(111)$ substrate). This is roughly double that for the single trans-azobenzene in the pore, $E_{\text {sub+trans }}-E_{\text {sub }}-E_{\text {trans }}=-1.51(-1.49) \mathrm{eV}$, and the small difference indicates the presence of an interaction between the molecules. The latter is quantified, and $E_{\text {sub+T+TR }}$ $-E_{\text {sub }+\mathrm{T}}-E_{\text {sub }+\mathrm{TR}}+E_{\text {sub }}=-0.08(-0.10) \mathrm{eV}$ is found, and the negative sign means an attractive molecule-molecule interaction. Note that these values correspond to about $5-7 \%$ of the adsorption energy of a single trans-azobenzene in the pore. More details and discussion on the hierarchy of interaction energies of the trans(rot)-trans configuration shown in Figure $8 \mathrm{~A}$ are reported in Section S3 of the Supporting Information.

The molecules (trans on the right side of Figure $8 \mathrm{~A}$, and trans(rot) on the left side of Figure $8 \mathrm{~A}$ ) are characterized by the following element-specific effective Bader charges $\left(Z_{\text {valence }}\right.$ - $Q_{\text {Bader }}$ ) averaged to one atom without (with) dipole correction: trans-N: $-0.53(-0.46)$ lel; trans-C: -0.03 $(+0.01)$ lel; trans-H: $+0.13(+0.07)$ lel; $\operatorname{trans}(\operatorname{rot})-\mathrm{N}:-0.47$ (-0.46) lel; trans(rot)-C: -0.05 (0.00) lel; and trans(rot)-H: $+0.15(+0.08)$ lel. These values are apparently very similar to those obtained for single azobenzene adsorption on $h$-BN/ $\mathrm{Rh}(111)$. Detailed data on the Bader charges of the constituting atoms of the two azobenzene molecules shown in Figure 8A are reported in Section S4 of the Supporting Information.

Given the energetically favored character of the trans(rot)trans configuration shown in Figure $8 \mathrm{~A}$ of two azobenzene molecules on the $\mathrm{h}-\mathrm{BN} / \mathrm{Rh}(111)$ substrate among the eight considered configurations reported in Figure 8, and the identified presence of an attractive molecule-molecule interaction, the formation of molecular chains shown in Figure $6 \mathrm{E}$ is possible at favorable conditions.

In the following, we investigate whether the moleculemolecule interaction can be increased further and how the adsorption and interaction energetics of two trans-azobenzene molecules above each other are related to those of a single trans-azobenzene by the template effect of the h-BN nanomesh on $\mathrm{Rh}(111)$. For this reason, we consider the trans(rot)-abovetrans (shown in Figure $8 \mathrm{~F}$ ) and the trans-above-trans (shown in Figure $8 \mathrm{G}$ ) configurations. We find that the moleculemolecule interactions are much larger for both of these configurations than for the energetically preferred trans(rot)trans with $-0.08(-0.10) \mathrm{eV}: E_{\text {sub }+\mathrm{T}+\mathrm{TR}}-E_{\text {sub }+\mathrm{T}}-E_{\text {sub+TR }}+$ $E_{\text {sub }}=-0.49(-0.47) \mathrm{eV}$ for the trans(rot)-above-trans and $E_{\text {sub }+\mathrm{T} 1+\mathrm{T} 2}-E_{\text {sub }+\mathrm{T} 1}-E_{\text {sub }+\mathrm{T} 2}+E_{\text {sub }}=-0.38(-0.39) \mathrm{eV}$ for the trans-above-trans without (with) dipole correction. Again, the negative sign means an attractive molecule-molecule interaction, and the obtained values correspond to about $25 \%$ (trans-above-trans) and to about 32\% (trans(rot)-above-trans) of the adsorption energy of a single trans-azobenzene in the pore. This ratio increases to about 33\% (trans-above-trans) and to about $40 \%$ (trans(rot)-above-trans) in comparison to the adsorption energy of a single trans-azobenzene on the wire $(-1.17 \mathrm{eV}$ with dipole correction). This important finding indicates that molecule-molecule interactions are expected to play a crucial role in molecular growth at larger molecular exposures in direct competition with template-driven growth. Furthermore, we note that the absolute value of the calculated adsorption energy of the second molecule $(-0.65 \mathrm{eV})$ in the trans(rot)-above-trans configuration (Figure $8 \mathrm{~F}$ ) is in excellent agreement with the derived desorption activation energy of $0.62 \mathrm{eV}$ related to the $240 \mathrm{~K}$ peak of the TPD data (Redhead approximation), which most likely corresponds to the onset of the growth of the second molecular layer in the pore. More details and discussion on the hierarchy of interaction energies of the trans(rot)-above-trans configuration shown in Figure 8F are reported in Section S3 of the Supporting Information.

The molecules in the trans(rot)-above-trans configuration (trans and trans(rot): the molecule below and above, respectively, in Figure $8 \mathrm{~F}$ ) are characterized by the following element-specific effective Bader charges $\left(Z_{\text {valence }}-Q_{\text {Bader }}\right)$ averaged to one atom without (with) dipole correction: trans$\mathrm{N}:-0.51(-0.48)$ lel; trans-C: $-0.04(+0.02)$ lel; trans-H: $+0.14(+0.07)$ lel; trans(rot) $-\mathrm{N}:-0.48(-0.45)$ lel; $\operatorname{trans}(\operatorname{rot})-$ C: $-0.05(+0.01)$ lel; and trans(rot) $-\mathrm{H}:+0.16(+0.08)$ lel. These values are, again, very similar to those obtained for single azobenzene adsorption on $h-\mathrm{BN} / \mathrm{Rh}(111)$ and also to those of the trans(rot)-trans configuration. Detailed data on the Bader charges of the constituting atoms of the two azobenzene molecules shown in Figure $8 \mathrm{~F}$ are reported in Section S4 of the Supporting Information. The illustration of the $3 \mathrm{D}$ charge density rearrangements corresponding to the 
molecule - molecule interaction, $\rho_{\text {sub }+\mathrm{T}+\mathrm{TR}}-\rho_{\text {sub }+\mathrm{T}}-\rho_{\mathrm{sub}+\mathrm{TR}}+$ $\rho_{\text {sub }}$, is shown in Figure 9.

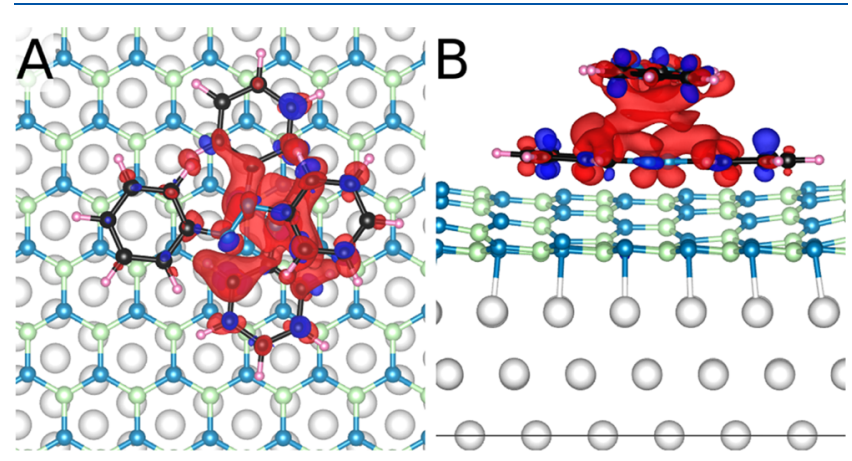

Figure 9. 3D charge transfers (A, top view; B, side view) representing the molecule-molecule interaction $\left(\rho_{\text {sub+T }+\mathrm{TR}}-\rho_{\mathrm{sub}+\mathrm{T}}-\rho_{\mathrm{sub}+\mathrm{TR}}+\right.$ $\rho_{\text {sub }}$ ) between two azobenzene molecules on the $\mathrm{h}-\mathrm{BN} / \mathrm{Rh}(111)$ substrate in the trans(rot)-above-trans configuration shown in Figure $8 \mathrm{~F}$ (isosurface value: $2 \times 10^{-4} \mathrm{lel} / \AA^{3}$; blue, electron accumulation; red, electron depletion). The color schemes for the atoms are reported in the caption of Figure 7.

Finally, related to STM experiments, we note that the main goal of Figure 6 is to prove the existence of the molecules in the pore regions of the $\mathrm{h}-\mathrm{BN} / \mathrm{Rh}(111)$ substrate. Going beyond that, and providing high-resolution simulated STM images, additional insight into the molecular orbital characters of the adsorbed molecules on the substrate is reported, which might serve as a useful reference for future high-resolution STM studies of azobenzene/h-BN/Rh(111) systems at low temperatures. Therefore, STM images are simulated for the energetically favored single cis- and trans- as well as the trans(rot)-trans-azobenzene adsorption configurations at opposite bias voltage polarities. The results for $\pm 1.5 \mathrm{~V}$ bias voltages are shown in Figure 10. As can be seen, the presence

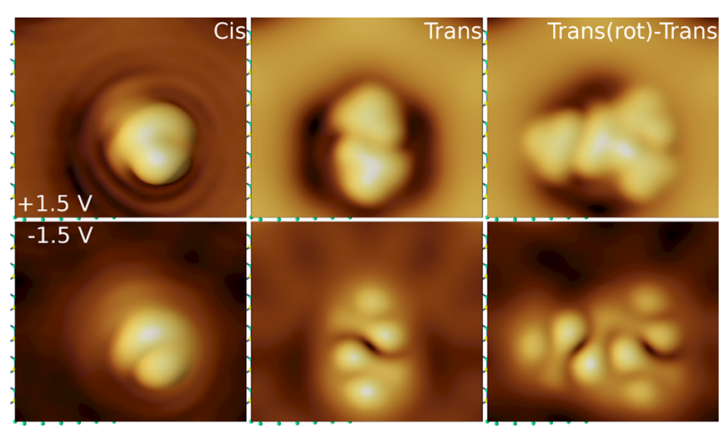

Figure 10. Simulated STM images of the energetically favored adsorption configurations of single azobenzene molecules (cis and trans in Figure 7) and of two azobenzene molecules (trans(rot)-trans in Figure 8A) in the pore of the h-BN/ $\mathrm{Rh}(111)$ substrate at opposite bias polarities (top and bottom). The size of the images is $3.2 \times 2.8$ $\mathrm{nm}^{2}$. Note that the bias voltage is applied on the sample as in the experimentally used definition.

of the molecules can be well recognized in the pore of the h$\mathrm{BN} / \mathrm{Rh}(111)$ nanomesh (particularly at positive bias voltage), just as in the experimental images in Figure 6. Furthermore, high-resolution imaging, as shown in Figure 10, provides the possibility of identifying the types and number of azobenzene molecules. Changing the bias polarity results in distinctly different molecular orbital characteristics for the (flat) trans- azobenzenes, while the cis-azobenzene does not show any substantial differences in the STM contrast. Additionally simulated bias-voltage-dependent STM images for the single trans-azobenzene molecule are reported in Section S5 of the Supporting Information.

\section{CONCLUSIONS}

The energetic and geometry aspects of the adsorption of azobenzene on h-BN/Rh(111) were addressed. TPD measurements disclosed that azobenzene desorption is characterized by two peaks in the submonolayer regime, tentatively assigned to molecules bound to the wire and pore regions. According to HREELS measurements, azobenzene adsorbs intact on the nitride surface at room temperature, with the molecular plane parallel to the surface. STM experiments revealed the templating effect of the periodically corrugated nanomesh: a strong preference for adsorption in the pores at $\sim 320 \mathrm{~K}$; however, in some cases, one-dimensional molecular stripes were also found, implying attractive molecule-molecule interactions. DFT calculations confirmed the experimental findings and provided more details about the adsorption energetics and bonding. Moreover, the calculations proved that azobenzene molecules keep their trans conformation also in the adsorbed phase. Simulated STM images indicated substantially different contrasts for the cis and trans forms.

Based on our results, we propose the following mechanism for azobenzene molecular growth on the $\mathrm{h}-\mathrm{BN} / \mathrm{Rh}(111)$ substrate. The trans-azobenzene is preferred compared to the cis isomer. At low amounts of the molecule, adsorption in the pore is clearly preferred, followed by wall- and wire-adsorption, showing a template effect. We identified a sizable moleculemolecule interaction for a $90^{\circ}$-rotated second trans-azobenzene molecule on the first one. Therefore, at larger amounts of azobenzene, the growth of the multimolecular structure is determined by the complex interplay between template-driven and molecule-molecule interactions. At certain conditions, the formation of the second molecular layer is expected concomitantly filling the wall and wire regions of the nanomesh. This mechanism could be engineered in the future by fine-tuning relevant growth parameters for arriving at a desired molecular structure. The present results may also serve as a basis for future studies related to the light-induced switching of azobenzene on h-BN, relevant for nanoelectronic applications.

\section{ASSOCIATED CONTENT}

\section{Supporting Information}

The Supporting Information is available free of charge at https://pubs.acs.org/doi/10.1021/acs.jpcc.0c01725.

Energetic contributions to the bonding of one and two azobenzene molecules on $\mathrm{h}-\mathrm{BN} / \mathrm{Rh}(111)$ for four configurations; details of Bader charge analysis; simulated STM images of the trans-azobenzene at different bias voltages; energetic analysis of TPD data compared with DFT (PDF)

\section{AUTHOR INFORMATION}

\section{Corresponding Authors}

L. Óvári - ELI-ALPS, ELI-HU Non-Profit Ltd., H-6728 Szeged, Hungary; MTA-SZTE Reaction Kinetics and Surface Chemistry Research Group, University of Szeged, H-6720 Szeged, 
Hungary; 이이이.org/0000-0001-8991-2708;

Email: ovari@chem.u-szeged.hu

K. Palotás - MTA-SZTE Reaction Kinetics and Surface Chemistry Research Group, University of Szeged, H-6720 Szeged, Hungary; Institute for Solid State Physics and Optics, Wigner Research Center for Physics, H-1525 Budapest, Hungary; (1) orcid.org/0000-0002-1914-2901;

Email: palotas.krisztian@wigner.hu

\section{Authors}

A. Szitás - Department of Applied and Environmental Chemistry, University of Szeged, H-6720 Szeged, Hungary

R. Gubó - Department of Applied and Environmental Chemistry, University of Szeged, H-6720 Szeged, Hungary; ELIALPS, ELI-HU Non-Profit Ltd., H-6728 Szeged, Hungary

T. Pásztor - MTA-SZTE Reaction Kinetics and Surface Chemistry Research Group, University of Szeged, H-6720 Szeged, Hungary

A. P. Farkas - ELI-ALPS, ELI-HU Non-Profit Ltd., H-6728 Szeged, Hungary; MTA-SZTE Reaction Kinetics and Surface Chemistry Research Group, University of Szeged, H-6720 Szeged, Hungary

T. Ajtai - ELI-ALPS, ELI-HU Non-Profit Ltd., H-6728 Szeged, Hungary; Department of Optics and Quantum Electronics, University of Szeged, H-6720 Szeged, Hungary

A. Berkó - MTA-SZTE Reaction Kinetics and Surface Chemistry Research Group, University of Szeged, H-6720 Szeged, Hungary

Z. Kónya - Department of Applied and Environmental Chemistry and MTA-SZTE Reaction Kinetics and Surface Chemistry Research Group, University of Szeged, H-6720 Szeged, Hungary; 이이이.org/0000-0002-9406-8596

Complete contact information is available at: https://pubs.acs.org/10.1021/acs.jpcc.0c01725

\section{Notes}

The authors declare no competing financial interest.

\section{ACKNOWLEDGMENTS}

Financial supports from the National Research Development and Innovation Office of Hungary projects No. K120115 and No. FK124100 are gratefully acknowledged. This research was also supported by the European Union and the State of Hungary, cofinanced by the European Social Fund in the framework of TÁMOP-4.2.4.A/2-11/1-2012-0001 "National Excellence Program". The ELI-ALPS project (GINOP-2.3.615-2015-00001) was supported by the European Union and cofinanced by the European Regional Development Fund. K.P. thanks E. Bruyer for useful discussions.

\section{REFERENCES}

(1) Novoselov, K. S.; Geim, A. K.; Morozov, S. V.; Jiang, D.; Zhang, Y.; Dubonos, S. V.; Grigorieva, I. V.; Firsov, A. A. Electric Field Effect in Atomically Thin Carbon Films. Science 2004, 306, 666-669.

(2) Novoselov, K. S.; Geim, A. K.; Morozov, S. V.; Jiang, D.; Katsnelson, M. I.; Grigorieva, I. V.; Dubonos, S. V.; Firsov, A. A. TwoDimensional Gas of Massless Dirac Fermions in Graphene. Nature 2005, 438, 197-200.

(3) Batzill, M. The Surface Science of Graphene: Metal Interfaces, CVD Synthesis, Nanoribbons, Chemical Modifications, and Defects. Surf. Sci. Rep. 2012, 67, 83-115.

(4) Agnoli, S.; Granozzi, G. Second Generation Graphene: Opportunities and Challenges for Surface Science. Surf. Sci. 2013, $609,1-5$.
(5) Xu, M.; Liang, T.; Shi, M.; Chen, H. Graphene-Like TwoDimensional Materials. Chem. Rev. 2013, 113, 3766-3798.

(6) Pakdel, A.; Bando, Y.; Golberg, D. Nano Boron Nitride Flatland. Chem. Soc. Rev. 2014, 43, 934-959.

(7) Auwärter, W. Hexagonal Boron Nitride Monolayers on Metal Supports: Versatile Templates for Atoms, Molecules and Nanostructures. Surf. Sci. Rep. 2019, 74, 1-95.

(8) Lindsay, L.; Broido, D. A. Enhanced Thermal Conductivity and Isotope Effect in Single-Layer Hexagonal Boron Nitride. Phys. Rev. B 2011, 84, No. 155421

(9) Yankowitz, M.; Xue, J.; LeRoy, B. J. Graphene on Hexagonal Boron Nitride. J. Phys.: Condens. Matter 2014, 26, No. 303201.

(10) Turner, M.; Golovko, V. B.; Vaughan, O. P. H.; Abdulkin, P.; Berenguer-Murcia, A.; Tikhov, M. S.; Johnson, B. F. G.; Lambert, R. M. Selective Oxidation with Dioxygen by Gold Nanoparticle Catalysts Derived from 55-Atom Clusters. Nature 2008, 454, 981-983.

(11) Grant, J. T.; Carrero, C. A.; Goeltl, F.; Venegas, J.; Mueller, P.; Burt, S. P.; Specht, S. E.; McDermott, W. P.; Chieregato, A.; Hermans, I. Selective Oxidative Dehydrogenation of Propane to Propene Using Boron Nitride Catalysts. Science 2016, 354, 15701573.

(12) Shi, L.; Wang, D.; Song, W.; Shao, D.; Zhang, W.-P.; Lu, A.-H. Edge-Hydroxylated Boron Nitride for Oxidative Dehydrogenation of Propane to Propylene. ChemCatChem 2017, 9, 1720.

(13) Paffett, M. T.; Simonson, R. J.; Papin, P.; Paine, R. T. Borazine Adsorption and Decomposition at $\operatorname{Pt}(111)$ and $\mathrm{Ru}(001)$ Surfaces. Surf. Sci. 1990, 232, 286-296.

(14) Nagashima, A.; Tejima, N.; Gamou, Y.; Kawai, T.; Oshima, C. Electronic Dispersion Relations of Monolayer Hexagonal Boron Nitride Formed on the Ni(111) Surface. Phys. Rev. B 1995, 51, 46064613.

(15) Morscher, M.; Corso, M.; Greber, T.; Osterwalder, J. Formation of Single Layer H-BN on Pd(111). Surf. Sci. 2006, 600, 3280-3284.

(16) Goriachko, A.; He, Y.; Knapp, M.; Over, H.; Corso, M.; Brugger, T.; Berner, S.; Osterwalder, J.; Greber, T. Self-Assembly of a Hexagonal Boron Nitride Nanomesh on $\mathrm{Ru}(0001)$. Langmuir 2007, 23, 2928-2931.

(17) Preobrajenski, A. B.; Nesterov, M. A.; Ng, M. L.; Vinogradov, A. S.; Mårtensson, N. Monolayer H-BN on Lattice-Mismatched Metal Surfaces: On the Formation of the Nanomesh. Chem. Phys. Lett. 2007, 446, 119-123.

(18) Ćavar, E.; Westerström, R.; Mikkelsen, A.; Lundgren, E.; Vinogradov, A. S.; Ng, M. L.; Preobrajenski, A. B.; Zakharov, A. A.; Mårtensson, N. A Single H-BN Layer on Pt(111). Surf. Sci. 2008, 602, $1722-1726$.

(19) Dong, G.; Fourré, E. B.; Tabak, F. C.; Frenken, J. W. M. How Boron Nitride Forms a Regular Nanomesh on Rh(111). Phys. Rev. Lett. 2010, 104, No. 096102.

(20) Orlando, F.; Larciprete, R.; Lacovig, P.; Boscarato, I.; Baraldi, A.; Lizzit, S. Epitaxial Growth of Hexagonal Boron Nitride on $\operatorname{Ir}(111)$. J. Phys. Chem. C 2012, 116, 157-164.

(21) Späth, F.; Gebhardt, J.; Düll, F.; Bauer, U.; Bachmann, P.; Gleichweit, C.; Görling, A.; Steinrück, H.-P.; Papp, C. Hydrogenation and Hydrogen Intercalation of Hexagonal Boron Nitride on $\mathrm{Ni}(11$ 1): Reactivity and Electronic Structure. 2D Mater. 2017, 4, No. 035026.

(22) Schwarz, M.; Riss, A.; Garnica, M.; Ducke, J.; Deimel, P. S.; Duncan, D. A.; Thakur, P. K.; Lee, T.-L.; Seitsonen, A. P.; Barth, J. V.; et al. Corrugation in the Weakly Interacting Hexagonal-BN/Cu(111) System: Structure Determination by Combining Noncontact Atomic Force Microscopy and X-Ray Standing Waves. ACS Nano 2017, 11, 9151-9161.

(23) Corso, M.; Greber, T.; Osterwalder, J. H-BN on Pd(1 10$)$ : A Tunable System for Self-Assembled Nanostructures? Surf. Sci. 2005, 577, L78-L84.

(24) Vinogradov, N. A.; Zakharov, A. A.; Ng, M. L.; Mikkelsen, A.; Lundgren, E.; Mårtensson, N.; Preobrajenski, A. B. One-Dimensional 
Corrugation of the h-BN Monolayer on Fe(110). Langmuir 2012, 28, $1775-1781$.

(25) Joshi, S.; Ecija, D.; Koitz, R.; Iannuzzi, M.; Seitsonen, A. P.; Hutter, J.; Sachdev, H.; Vijayaraghavan, S.; Bischoff, F.; Seufert, K.; et al. Boron Nitride on $\mathrm{Cu}(111)$ : An Electronically Corrugated Monolayer. Nano Lett. 2012, 12, 5821-5828.

(26) Müller, F.; Hüfner, S.; Sachdev, H.; Laskowski, R.; Blaha, P.; Schwarz, K. Epitaxial Growth of Hexagonal Boron Nitride on Ag(111). Phys. Rev. B 2010, 82, No. 113406.

(27) Laskowski, R.; Blaha, P.; Schwarz, K. Bonding of Hexagonal BN to Transition Metal Surfaces: An Ab Initio Density-Functional Theory Study. Phys. Rev. B 2008, 78, No. 045409.

(28) Díaz, J. G.; Ding, Y.; Koitz, R.; Seitsonen, A. P.; Iannuzzi, M.; Hutter, J. Hexagonal Boron Nitride on Transition Metal Surfaces. Theor. Chem. Acc. 2013, 132, No. 1350.

(29) Camilli, L.; Sutter, E.; Sutter, P. Growth of Two-Dimensional Materials on Non-Catalytic Substrates: H-BN/Au(111). 2D Mater. 2014, 1, No. 025003.

(30) Brihuega, I.; Michaelis, C. H.; Zhang, J.; Bose, S.; Sessi, V.; Honolka, J.; Alexander Schneider, M.; Enders, A.; Kern, K. Electronic Decoupling and Templating of Co Nanocluster Arrays on the Boron Nitride Nanomesh. Surf. Sci. 2008, 602, L95-L99.

(31) Laskowski, R.; Blaha, P. Unraveling the Structure of the H-BN/ $\mathrm{Rh}(111)$ Nanomesh with Ab Initio Calculations. J. Phys.: Condens. Matter 2008, 20, No. 064207.

(32) Berner, S.; Corso, M.; Widmer, R.; Groening, O.; Laskowski, R.; Blaha, P.; Schwarz, K.; Goriachko, A.; Over, H.; Gsell, S.; et al. Boron Nitride Nanomesh: Functionality from a Corrugated Monolayer. Angew. Chem., Int. Ed. 2007, 46, 5115-5119.

(33) Gubó, R.; Vári, G.; Kiss, J.; Farkas, A. P.; Palotás, K.; Óvári, L.; Berkó, A.; Kónya, Z. Tailoring the Hexagonal Boron Nitride Nanomesh on Rh(111) with Gold. Phys. Chem. Chem. Phys. 2018, 20, 15473-15485.

(34) Patterson, M. C.; Habenicht, B. F.; Kurtz, R. L.; Liu, L.; Xu, Y.; Sprunger, P. T. Formation and Stability of Dense Arrays of $\mathrm{Au}$ Nanoclusters on Hexagonal Boron Nitride/Rh(111). Phys. Rev. B: Condens. Matter Mater. Phys. 2014, 89, No. 205423.

(35) Düll, F.; Freiberger, E. M.; Bachmann, P.; Steinhauer, J.; Papp, C. Pt Nanoclusters Sandwiched between Hexagonal Boron Nitride and Nanographene as van Der Waals Heterostructures for Optoelectronics. ACS Appl. Nano Mater. 2019, 2, 7019-7024.

(36) Corso, M.; Auwärter, W.; Muntwiler, M.; Tamai, A.; Greber, T.; Osterwalder, J. Boron Nitride Nanomesh. Science 2004, 303, $217-$ 220.

(37) Dil, H.; Lobo-Checa, J.; Laskowski, R.; Blaha, P.; Berner, S.; Osterwalder, J.; Greber, T. Surface Trapping of Atoms and Molecules with Dipole Rings. Science 2008, 319, 1824-1826.

(38) Iannuzzi, M.; Tran, F.; Widmer, R.; Dienel, T.; Radican, K.; Ding, Y.; Hutter, J.; Gröning, O. Site-Selective Adsorption of Phthalocyanine on h-BN/Rh(111) Nanomesh. Phys. Chem. Chem. Phys. 2014, 16, 12374-12384.

(39) Liu, L.; Dienel, T.; Widmer, R.; Gröning, O. Interplay between Energy-Level Position and Charging Effect of Manganese Phthalocyanines on an Atomically Thin Insulator. ACS Nano 2015, 9, $10125-10132$.

(40) Farkas, A. P.; Szitás, Á.; Vári, G.; Gubó, R.; Óvári, L.; Berkó, A.; Kiss, J.; Kónya, Z. Effect of Gold on the Adsorption Properties of Acetaldehyde on Clean and H-BN Covered Rh (111) Surface. Top. Catal. 2018, 61, 1247-1256.

(41) Óvári, L.; Wolf, M.; Tegeder, P. Reversible Changes in the Vibrational Structure of Tetra- Tert -Butylazobenzene on a Au (111) Surface Induced by Light and Thermal Activation. J. Phys. Chem. C 2007, 111, 15370-15374.

(42) Tegeder, P. Optically and Thermally Induced Molecular Switching Processes at Metal Surfaces. J. Phys.: Condens. Matter 2012, 24, No. 394001.

(43) Bronner, C.; Schulze, M.; Hagen, S.; Tegeder, P. The Influence of the Electronic Structure of Adsorbate-Substrate Complexes on Photoisomerization Ability. New J. Phys. 2012, 14, No. 043023.
(44) Schulze, M.; Bronner, C.; Tegeder, P. Adsorption Energetics of Azobenzenes on Noble Metal Surfaces. J. Phys.: Condens. Matter 2014, 26, No. 355004.

(45) Tsuji, T.; Takashima, H.; Takeuchi, H.; Egawa, T.; Konaka, S. Molecular Structure and Torsional Potential of Trans -Azobenzene. A Gas Electron Diffraction Study. J. Phys. Chem. A 2001, 105, 93479353.

(46) Armstrong, D. R.; Clarkson, J.; Smith, W. E. Vibrational Analysis of Trans-Azobenzene. J. Phys. Chem. A 1995, 99, 1782517831.

(47) Biswas, N.; Umapathy, S. Density Functional Calculations of Structures, Vibrational Frequencies, and Normal Modes of Trans and Cis -Azobenzene. J. Phys. Chem. A 1997, 101, 5555-5566.

(48) Kurita, N.; Tanaka, S.; Itoh, S. Ab Initio Molecular Orbital and Density Functional Studies on the Stable Structures and Vibrational Properties of Trans - and Cis -Azobenzenes. J. Phys. Chem. A 2000, 104, 8114-8120.

(49) Fliegl, H.; Köhn, A.; Hättig, C.; Ahlrichs, R. Ab Initio Calculation of the Vibrational and Electronic Spectra of Trans - and Cis -Azobenzene. J. Am. Chem. Soc. 2003, 125, 9821-9827.

(50) Briquet, L.; Vercauteren, D. P.; Perpète, E. A.; Jacquemin, D. Is Solvated Trans-Azobenzene Twisted or Planar? Chem. Phys. Lett. 2006, 417, 190-195.

(51) Duarte, L.; Fausto, R.; Reva, I. Structural and Spectroscopic Characterization of E- and Z-Isomers of Azobenzene. Phys. Chem. Chem. Phys. 2014, 16, 16919-16930.

(52) Lin, M. M.; Shorokhov, D.; Zewail, A. H. Conformations and Coherences in Structure Determination by Ultrafast Electron Diffraction. J. Phys. Chem. A 2009, 113, 4075-4093.

(53) Kresse, G.; Furthmüller, J. Efficient Iterative Schemes for Ab Initio Total-Energy Calculations Using a Plane-Wave Basis Set. Phys. Rev. B 1996, 54, 11169-11186.

(54) Kresse, G.; Furthmüller, J. Efficiency of Ab-Initio Total Energy Calculations for Metals and Semiconductors Using a Plane-Wave Basis Set. Comput. Mater. Sci. 1996, 6, 15-50.

(55) Kresse, G.; Joubert, D. From Ultrasoft Pseudopotentials to the Projector Augmented-Wave Method. Phys. Rev. B 1999, 59, 17581775.

(56) Klimeš, J.; Bowler, D. R.; Michaelides, A. Chemical Accuracy for the van Der Waals Density Functional. J. Phys.: Condens. Matter 2010, 22, No. 022201.

(57) Klimeš, J.; Bowler, D. R.; Michaelides, A. Van Der Waals Density Functionals Applied to Solids. Phys. Rev. B 2011, 83, No. 195131.

(58) Neugebauer, J.; Scheffler, M. Adsorbate-Substrate and Adsorbate-Adsorbate Interactions of $\mathrm{Na}$ and $\mathrm{K}$ Adlayers on Al(111). Phys. Rev. B 1992, 46, 16067-16080.

(59) Henkelman, G.; Arnaldsson, A.; Jónsson, H. A Fast and Robust Algorithm for Bader Decomposition of Charge Density. Comput. Mater. Sci. 2006, 36, 354-360.

(60) Sanville, E.; Kenny, S. D.; Smith, R.; Henkelman, G. Improved Grid-Based Algorithm for Bader Charge Allocation. J. Comput. Chem. 2007, 28, 899.

(61) Tang, W.; Sanville, E.; Henkelman, G. A Grid-Based Bader Analysis Algorithm without Lattice Bias. J. Phys.: Condens. Matter 2009, 21, No. 084204.

(62) Mándi, G.; Palotás, K. Chen's Derivative Rule Revisited: Role of Tip-Orbital Interference in STM. Phys. Rev. B 2015, 91, No. 165406.

(63) Hofer, W. Challenges and Errors: Interpreting High Resolution Images in Scanning Tunneling Microscopy. Prog. Surf. Sci. 2003, 71, 147-183.

(64) Palotás, K.; Hofer, W. A. Multiple Scattering in a Vacuum Barrier Obtained from Real-Space Wavefunctions. J. Phys.: Condens. Matter 2005, 17, 2705-2713.

(65) King, D. A. Thermal Desorption from Metal Surfaces: A Review. Surf. Sci. 1975, 47, 384-402.

(66) Rokuta, E.; Hasegawa, Y.; Suzuki, K.; Gamou, Y.; Oshima, C.; Nagashima, A. Phonon Dispersion of an Epitaxial Monolayer Film of 
Hexagonal Boron Nitride on Ni(111). Phys. Rev. Lett. 1997, 79, 4609-4612.

(67) Ibach, H.; Mills, D. L. Electron Energy Loss Spectroscopy and Surface Vibrations; Academic Press: New York, 1982.

(68) Farkas, A. P.; Szitás, Á.; Jurdi, D.; Palotás, K.; Kiss, J.; Kónya, Z. Selective Transformation of Ethanol to Acetaldehyde Catalyzed by $\mathrm{Au} / \mathrm{h}-\mathrm{BN}$ Interface Prepared on Rh (111) Surface. Appl. Catal., A 2020, 592, No. 117440.

(69) Cabral, L.; Sabino, F. P.; Lima, M. P.; Marques, G. E.; LopezRichard, V.; Da Silva, J. L. F. Azobenzene Adsorption on the $\mathrm{MoS}_{2}(0001)$ Surface: A Density Functional Investigation within van Der Waals Corrections. J. Phys. Chem. C 2018, 122, 18895-18901.

(70) Fu, Q.; Cocchi, C.; Nabok, D.; Gulans, A.; Draxl, C. GrapheneModulated Photo-Absorption in Adsorbed Azobenzene Monolayers. Phys. Chem. Chem. Phys. 2017, 19, 6196-6205.

(71) Henningsen, N.; Rurali, R.; Franke, K. J.; Fernández-Torrente, I.; Pascual, J. I. Trans to Cis Isomerization of an Azobenzene Derivative on a $\mathrm{Cu}$ (100) Surface. Appl. Phys. A: Mater. Sci. Process. 2008, 93, No. 241. 